\title{
ICI-AWARE PARAMETER ESTIMATION FOR MIMO-OFDM RADAR VIA APES SPATIAL FILTERING
}

\author{
Musa Furkan Keskin*, Henk Wymeersch*, Visa Koivunen ${ }^{\dagger}$ \\ ${ }^{*}$ Chalmers University of Technology, Gothenburg, Sweden \\ ${ }^{\dagger}$ Aalto University, Espoo, Finland
}

\begin{abstract}
We propose a novel three-stage delay-Doppler-angle estimation algorithm for a MIMO-OFDM radar in the presence of inter-carrier interference (ICI). First, leveraging the observation that spatial covariance matrix is independent of target delays and Dopplers, we perform angle estimation via the MUSIC algorithm. For each estimated angle, we next formulate the radar delay-Doppler estimation as a joint carrier frequency offset (CFO) and channel estimation problem via an APES (amplitude and phase estimation) spatial filtering approach by transforming the delay-Doppler parameterized radar channel into an unstructured form. In the final stage, delay and Doppler of each target can be recovered from target-specific channel estimates over time and frequency. Simulation results illustrate the superior performance of the proposed algorithm in high-mobility scenarios.
\end{abstract}

Index Terms - OFDM, joint radar-communications, intercarrier interference, APES, CFO estimation.

\section{INTRODUCTION}

With a huge increase of interest in co-existence and spectrum sharing among radar and communications, joint radar-communications (JRC) strategies are being actively developed for $5 \mathrm{G}$ and beyond wireless systems [1-6]. A promising approach to practical JRC implementation is to design dual-functional radar-communications (DFRC) systems, which employ a single hardware that can simultaneously perform radar sensing and data transmission with a codesigned waveform [2, 7, 8]. Orthogonal frequency-division multiplexing (OFDM) has been widely investigated as a DFRC waveform due its wide availability in wireless communication systems and its potential to achieve high radar performance [9-11]. In the literature, estimator design for OFDM radar sensing has been studied in both single-antenna [9,11,12] and multiple-input multiple-output (MIMO) [13, 14] settings.

In high-mobility scenarios, such as millimeter-wave (mmWave) vehicular JRC systems [2], Doppler-induced intercarrier interference (ICI) can significantly degrade the performance of OFDM from both radar and communications perspective [15]17]. To improve OFDM radar performance, various ICI mitigation approaches have been proposed [11, 12, 15, 16]. In [15], the ICI effect is eliminated via an allcell Doppler correction (ACDC) method, which requires the OFDM symbol matrix to be rank-one and therefore leads to a substantial loss in data rate. Similarly, ICI mitigation technique in [16] imposes certain constraints on transmit symbols, which impedes dual-functional operation. An alternating maximization approach is designed in [11] to reduce the complexity of high-dimensional maximum-likelihood

This work is supported by Vinnova grant 2018-01929 and the Marie Skłodowska-Curie Individual Fellowships (H2020-MSCA-IF-2019) Grant 888913 (OTFS-RADCOM).
(ML) search by assuming that the number of targets is known apriori. The work in [12] considers a single-target scenario and proposes a pulse compression technique to compensate for ICI-induced phase rotations across OFDM subcarriers.

In this paper, we propose an ICI-aware delay-Doppler-angle estimation algorithm for a MIMO-OFDM radar with arbitrary transmit symbols in a generic multi-target scenario. The main ingredient is to re-formulate radar sensing as a joint carrier frequency offset $(\mathrm{CFO})^{1}$ and channel estimation problem, which allows us to decontaminate the ICI effect from the resulting channel estimates, leading to highaccuracy delay-Doppler estimation. To that end, we first perform angle estimation using the MUSIC high-resolution direction finding algorithm [18] based on the observation that spatial covariance matrix does not depend on target delays and Dopplers. To suppress mutual multi-target interferences [19] in the spatial domain, we then devise an APES-like spatial filtering approach [1,20] that performs joint Doppler/CFO and unstructured radar channel estimation for each estimated target angle separately. Finally, delay-Doppler of each target can be estimated from target-specific channel estimates by exploiting the OFDM time-frequency structure. Simulations are carried out to demonstrate the performance of the proposed algorithm in highmobility scenarios. To the best of authors' knowledge, this is the first algorithm for MIMO-OFDM radar that takes into account the effect of ICI in estimating multiple target parameters without imposing any structure on data symbols.

Notations: Uppercase (lowercase) boldface letters are used to denote matrices (vectors). $(\cdot)^{*},(\cdot)^{T}$ and $(\cdot)^{H}$ represent conjugate, transpose and Hermitian transpose operators, respectively. $\Re\{\cdot\}$ denotes the real part. The $n^{\text {th }}$ entry of a vector $\mathbf{x}$ is denoted as $[\mathbf{x}]_{i}$, while the $(m, n)^{\text {th }}$ element of a matrix $\mathbf{X}$ is $[\mathbf{X}]_{m, n} . \Pi_{\mathbf{X}}=$ $\mathbf{X}\left(\mathbf{X}^{H} \mathbf{X}\right)^{-1} \mathbf{X}^{H}$ represents the orthogonal projection operator onto the column space of $\mathbf{X}$ and $\odot$ denotes the Hadamard product.

\section{OFDM RADAR SYSTEM MODEL}

Consider an OFDM DFRC transceiver that communicates with an OFDM receiver while concurrently performing radar sensing using the backscattered signals for target detection [7, 9]. The DFRC transceiver is equipped with an $N_{\mathrm{T}}$-element transmit (TX) uniform linear array (ULA) and an $N_{\mathrm{R}}$-element receive (RX) ULA. We assume co-located and perfectly decoupled TX/RX antenna arrays so that the radar receiver does not suffer from self-interference due to full-duplex radar operation [9, 21-23]. In this section, we derive OFDM transmit and receive signal models and formulate the multitarget parameter estimation problem.

\subsection{Transmit Signal Model}

We consider an OFDM communication frame consisting of $M$ OFDM symbols, each of which has a total duration of $T_{\text {sym }}=$

\footnotetext{
${ }^{1}$ Borrowing from the OFDM communications literature [17], we use Doppler and CFO interchangeably throughout the text.
} 
$T_{\mathrm{cp}}+T$ and a total bandwidth of $N \Delta f=B$. Here, $T_{\mathrm{cp}}$ and $T$ denote, respectively, the cyclic prefix $(\mathrm{CP})$ duration and the elementary symbol duration, $\Delta f=1 / T$ is the subcarrier spacing, and $N$ is the number of subcarriers [9]. Then, the complex baseband transmit signal for the $m^{\text {th }}$ symbol is given by

$$
s_{m}(t)=\frac{1}{\sqrt{N}} \sum_{n=0}^{N-1} x_{n, m} e^{j 2 \pi n \Delta f t} \operatorname{rect}\left(\frac{t-m T_{\mathrm{sym}}}{T_{\mathrm{sym}}}\right)
$$

where $x_{n, m}$ denotes the complex data symbol on the $n^{\text {th }}$ subcarrier for the $m^{\text {th }}$ symbol [10], and rect $(t)$ is a rectangular function that takes the value 1 for $t \in[0,1]$ and 0 otherwise. Assuming a singlestream beamforming model [13, 23, 24], the transmitted signal over the block of $M$ symbols for $t \in\left[0, M T_{\text {sym }}\right]$ can be written as

$$
\Re\left\{\mathbf{f}_{\mathrm{T}} \sum_{m=0}^{M-1} s_{m}(t) e^{j 2 \pi f_{c} t}\right\}
$$

where $f_{c}$ and $\mathbf{f}_{\mathrm{T}} \in \mathbb{C}^{N_{\mathrm{T}} \times 1}$ denote, respectively, the carrier frequency and the TX beamforming vector.

\subsection{Receive Signal Model}

Suppose there exists a point target in the far-field, characterized by a complex channel gain $\alpha$ (including path loss and radar cross section effects), an azimuth angle $\theta$, a round-trip delay $\tau$ and a normalized Doppler shift $\nu=2 v / c$ (leading to a time-varying delay $\tau(t)=\tau-\nu t$ ), where $v$ and $c$ denote the radial velocity and speed of propagation, respectively. In addition, let $\mathbf{a}_{\mathrm{T}}(\theta) \in \mathbb{C}^{N_{\mathrm{T}} \times 1}$ and $\mathbf{a}_{\mathrm{R}}(\theta) \in \mathbb{C}^{N_{\mathrm{R}} \times 1}$ denote, respectively, the steering vectors of the TX and RX ULAs, i.e., $\left[\mathbf{a}_{\mathrm{T}}(\theta)\right]_{i}=e^{j \frac{2 \pi}{\lambda} d i \sin (\theta)}$ and $\left[\mathbf{a}_{\mathrm{R}}(\theta)\right]_{i}=$ $e^{j \frac{2 \pi}{\lambda} d i \sin (\theta)}$, where $\lambda$ and $d=\lambda / 2$ denote the signal wavelength and antenna element spacing, respectively. Given the transmit signal model in (2), the backscattered signal impinging onto the $i^{\text {th }}$ element of the radar RX array can be expressed as

$y_{i}(t)=\alpha\left[\mathbf{a}_{\mathrm{R}}(\theta)\right]_{i} \mathbf{a}_{\mathrm{T}}^{T}(\theta) \mathbf{f}_{\mathrm{T}} \sum_{m=0}^{M-1} s_{m}(t-\tau(t)) e^{-j 2 \pi f_{c} \tau} e^{j 2 \pi f_{c} \nu t}$.

We make the following standard assumptions: (i) the CP duration is larger than the round-trip delay of the furthermost target ${ }^{2}$, i.e., $T_{\mathrm{cp}} \geq \tau$, [2]12, 22], (ii) the Doppler shifts satisfy $|\nu| \ll 1 / N$ [11 12], and (iii) the time-bandwidth product (TBP) $B M T_{\mathrm{sym}}$ is sufficiently low so that the wideband effect can be ignored, i.e., $s_{m}(t-\tau(t)) \approx s_{m}(t-\tau)$ [15]. Under this setting, sampling $y_{i}(t)$ at $t=m T_{\mathrm{sym}}+T_{\mathrm{cp}}+\ell T / N$ for $\ell=0, \ldots, N-1$ (i.e., after $\mathrm{CP}$ removal for the $m^{\text {th }}$ symbol) and neglecting constant terms, the time-domain signal received by the $i^{\text {th }}$ antenna in the $m^{\text {th }}$ symbol can be written as [11]

$$
\begin{aligned}
y_{i, m}[\ell]= & \alpha\left[\mathbf{a}_{\mathrm{R}}(\theta)\right]_{i} \mathbf{a}_{\mathrm{T}}^{T}(\theta) \mathbf{f}_{\mathrm{T}} e^{j 2 \pi f_{c} m T_{\mathrm{sym}} \nu} e^{j 2 \pi f_{c} T \frac{\ell}{N} \nu} \\
& \times \frac{1}{\sqrt{N}} \sum_{n=0}^{N-1} x_{n, m} e^{j 2 \pi n \frac{\ell}{N}} e^{-j 2 \pi n \Delta f \tau} .
\end{aligned}
$$

\subsection{Fast-Time/Slow-Time Representation with ICI}

For the sake of convenience, let us define, respectively, the frequencydomain and temporal steering vectors and the ICI phase rotation

\footnotetext{
${ }^{2}$ We focus on small surveillance volumes where the targets are relatively close to the radar, such as vehicular applications.
}

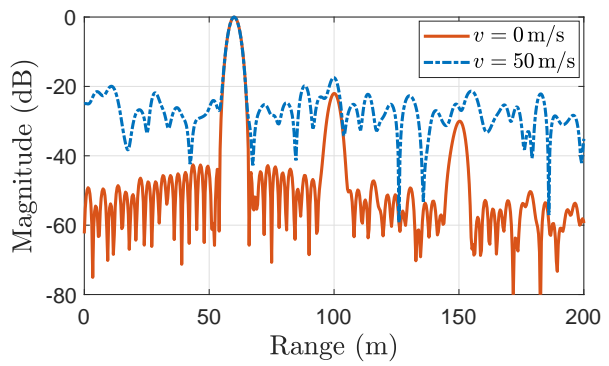

Fig. 1. Range profiles of MIMO-OFDM radar with the parameters given in Sec. 4 for two different target velocities. The scenario contains 3 targets having the same velocity $v$, the ranges $(60,100,150) \mathrm{m}$, the angles $\left(25^{\circ}, 30^{\circ}, 35^{\circ}\right)$ and the SNRs (i.e., $\left.\left|\alpha_{k}\right|^{2} / \sigma^{2}\right)(30,5,0) \mathrm{dB}$, respectively. matrix as

$$
\begin{aligned}
\mathbf{b}(\tau) \triangleq\left[1, e^{-j 2 \pi \Delta f \tau}, \ldots, e^{-j 2 \pi(N-1) \Delta f \tau}\right]^{T} \\
\mathbf{c}(\nu) \triangleq\left[1, e^{-j 2 \pi f_{c} T_{\mathrm{sym}} \nu}, \ldots, e^{-j 2 \pi f_{c}(M-1) T_{\mathrm{sym}} \nu}\right]^{T} \\
\mathbf{D}(\nu) \triangleq \operatorname{diag}\left(1, e^{j 2 \pi f_{c} \frac{T}{N} \nu}, \ldots, e^{j 2 \pi f_{c} \frac{T(N-1)}{N} \nu}\right)
\end{aligned}
$$

Accordingly, the radar observations in (3) can be expressed as

$$
\mathbf{y}_{i, m}=\alpha\left[\mathbf{a}_{\mathrm{R}}(\theta)\right]_{i} \mathbf{a}_{\mathrm{T}}^{T}(\theta) \mathbf{f}_{\mathrm{T}} \mathbf{D}(\nu) \mathbf{F}_{N}^{H}\left(\mathbf{x}_{m} \odot \mathbf{b}(\tau)\left[\mathbf{c}^{*}(\nu)\right]_{m}\right)
$$

where $\mathbf{F}_{N} \in \mathbb{C}^{N \times N}$ is the unitary DFT matrix with $[\mathbf{F}]_{\ell, n}=$ $\frac{1}{\sqrt{N}} e^{-j 2 \pi n \frac{\ell}{N}}, \mathbf{y}_{i, m} \triangleq\left[y_{i, m}[0] \ldots y_{i, m}[N-1]\right]^{T}$ and $\mathbf{x}_{m} \triangleq$ $\left[x_{0, m} \ldots x_{N-1, m}\right]^{T}$.

Aggregating (7) over $M$ symbols and considering the presence of multiple targets, the OFDM radar signal received by the $i^{\text {th }}$ antenna over a frame can be written in a fast-time/slow-time compact matrix form as

$$
\mathbf{Y}_{i}=\sum_{k=0}^{K-1} \alpha_{k}^{(i)} \underbrace{\mathbf{D}\left(\nu_{k}\right)}_{\text {ICI }} \mathbf{F}_{N}^{H}\left(\mathbf{X} \odot \mathbf{b}\left(\tau_{k}\right) \mathbf{c}^{H}\left(\nu_{k}\right)\right)+\mathbf{Z}_{i}
$$

for $i=0, \ldots, N_{\mathrm{R}}-1$, where $\alpha_{k}^{(i)} \triangleq \alpha_{k}\left[\mathbf{a}_{\mathrm{R}}\left(\theta_{k}\right)\right]_{i} \mathbf{a}_{\mathrm{T}}^{T}\left(\theta_{k}\right) \mathbf{f}_{\mathrm{T}}$, $\left(\alpha_{k}, \tau_{k}, \nu_{k}, \theta_{k}\right)$ are the parameters of the $k^{\text {th }}$ target, $\mathbf{Y}_{i} \triangleq\left[\mathbf{y}_{i, 0} \ldots\right.$ $\left.\mathbf{y}_{i, M-1}\right] \in \mathbb{C}^{N \times M}, \mathbf{X} \triangleq\left[\mathbf{x}_{0} \ldots \mathbf{x}_{M-1}\right] \in \mathbb{C}^{N \times M}$ and $\mathbf{Z}_{i} \in$ $\mathbb{C}^{N \times M}$ is the additive noise matrix with $\operatorname{vec}\left(\mathbf{Z}_{i}\right) \sim \mathcal{C N}\left(\mathbf{0}_{N M}\right.$, $\left.\sigma^{2} \mathbf{I}_{N M}\right)$. In (8), each column contains fast-time samples within a particular symbol and each row contains slow-time samples at a particular range bin. The diagonal phase rotation matrix $\mathbf{D}(\nu)$ quantifies the ICI effect in fast-time domain, leading to Doppler-dependent phase-shifts across fast-time samples of each OFDM symbol, similar to the CFO effect in OFDM communications [25, 26]. Fig. 1 illustrates the effect of ICI on the range profile of an OFDM radar.

Given the transmit data symbols $\mathbf{X}$, the problem of interest for OFDM radar sensing is to estimate channel gains $\left\{\alpha_{k}\right\}_{k=0}^{K-1}$, azimuth angles $\left\{\theta_{k}\right\}_{k=0}^{K-1}$, delays $\left\{\tau_{k}\right\}_{k=0}^{K-1}$ and Doppler shifts $\left\{\nu_{k}\right\}_{k=0}^{K-1}$ from the received $N_{\mathrm{R}} \times N \times M$ space/fast-time/slow-time data cube $\left\{\mathbf{Y}_{i}\right\}_{i=0}^{N_{\mathrm{R}}-1}$ in 8 .

\section{PARAMETER ESTIMATION VIA APES SPATIAL FILTERING}

The ML approach to (8) requires a computationally prohibitive $3 K$ dimensional search over the parameter space. Additionally, the number of targets $K$ is unknown a-priori. To tackle this challenging estimation problem, we devise a three-stage low-complexity algorithm as described in the following subsections. 


\subsection{Step 1: Angle Estimation via MUSIC}

For mathematical convenience, we consider the space/fast-time snapshot of the data cube in (8) corresponding to the $m^{\text {th }}$ OFDM symbol:

$$
\begin{aligned}
\overline{\mathbf{Y}}_{m} \triangleq & {\left[\mathbf{y}_{0, m} \ldots \mathbf{y}_{N_{\mathrm{R}}-1, m}\right] \in \mathbb{C}^{N \times N_{\mathrm{R}}} } \\
= & \sum_{k=0}^{K-1} \alpha_{k} \mathbf{a}_{\mathrm{T}}^{T}\left(\theta_{k}\right) \mathbf{f}_{\mathrm{T}} \mathbf{D}\left(\nu_{k}\right) \mathbf{F}_{N}^{H} \operatorname{diag}\left(\mathbf{x}_{m}\right) \\
& \quad \times \mathbf{b}\left(\tau_{k}\right)\left[\mathbf{c}^{*}\left(\nu_{k}\right)\right]_{m} \mathbf{a}_{\mathrm{R}}^{T}\left(\theta_{k}\right)+\overline{\mathbf{Z}}_{m}
\end{aligned}
$$

for $m=0, \ldots, M-1$. We propose to perform angle estimation using the MUSIC algorithm [18]. To this end, we first construct the spatial covariance matrix (SCM) of the data cube in (9) as

$$
\mathbf{R} \triangleq \sum_{m=0}^{M-1} \overline{\mathbf{Y}}_{m}^{H} \overline{\mathbf{Y}}_{m}
$$

Under the assumption of spatially non-overlapping targets (i.e., $\left.\mathbf{a}_{\mathrm{R}}^{H}\left(\theta_{k_{1}}\right) \mathbf{a}_{\mathrm{R}}\left(\theta_{k_{2}}\right) \approx 0, \forall k_{1} \neq k_{2}\right)$, the SCM can be approximated as (the proof is omitted due to space limitation)

$$
\mathbf{R} \approx\|\mathbf{X}\|_{F}^{2} \sum_{k=0}^{K-1}\left|\alpha_{k}\right|^{2}\left|\mathbf{a}_{\mathrm{T}}^{T}\left(\theta_{k}\right) \mathbf{f}_{\mathrm{T}}\right|^{2} \mathbf{a}_{\mathrm{R}}^{*}\left(\theta_{k}\right) \mathbf{a}_{\mathrm{R}}^{T}\left(\theta_{k}\right)+\sigma^{2} \mathbf{I}
$$

which is independent of target delays and Dopplers. Hence, creating the MUSIC spectrum from the SCM in 10 does not require target delay-Doppler information. Assuming $N_{\mathrm{R}}>K$, let the eigendecomposition of the SCM be denoted as $\mathbf{R}=$ $\mathbf{U}_{s} \boldsymbol{\Lambda}_{s} \mathbf{U}_{s}^{H}+\mathbf{U}_{n} \boldsymbol{\Lambda}_{n} \mathbf{U}_{n}^{H}$, where the diagonal matrix $\boldsymbol{\Lambda}_{s}$ contains the $K$ largest eigenvalues, $\boldsymbol{\Lambda}_{n}$ contains the remaining $N_{\mathrm{R}}-K$ eigenvalues, and $\mathbf{U}_{s}$ and $\mathbf{U}_{n}$ have the corresponding eigenvectors as their columns. Then, the MUSIC spectrum can be computed as

$$
f(\theta)=\frac{1}{\mathbf{a}_{\mathrm{R}}^{T}(\theta) \mathbf{U}_{n} \mathbf{U}_{n}^{H} \mathbf{a}_{\mathrm{R}}^{*}(\theta)} .
$$

Let $\mathcal{S}=\left\{\widehat{\theta}_{0}, \ldots, \widehat{\theta}_{K-1}\right\}$ be the set of estimated angles in Step 1, which correspond to the peaks of the MUSIC spectrum ${ }^{3}$ in (12).

\subsection{Step 2: Joint Doppler/CFO and Unstructured Channel Es-} timation via APES Beamforming

In Step 2, we formulate a joint Doppler/CFO and channel estimation problem for each $\widehat{\theta} \in \mathcal{S}$, assuming the existence of a single target at a given angle. Invoking the assumption of spatially non-overlapping targets, we treat interferences from other target components as noise and consider a single-target model in 9 for each $\widehat{\theta} \in \mathcal{S}$. To that aim, let

$$
\mathbf{H}=\left[\mathbf{h}_{0} \ldots \mathbf{h}_{M-1}\right] \in \mathbb{C}^{L \times M}
$$

denote the unstructured, single-target radar channels in the time domain with $L$ taps, collected over $M$ OFDM symbols. Here, $L \leq$ $N T_{\mathrm{cp}} / T$ due to the $\mathrm{CP}$ requirement. Based on this unstructured representation, (9) can be re-written as

$$
\overline{\mathbf{Y}}_{m}=\mathbf{D}(\nu) \overline{\mathbf{X}}_{m} \mathbf{h}_{m} \mathbf{a}_{\mathrm{R}}^{T}(\widehat{\theta})+\overline{\mathbf{Z}}_{m}
$$

where $\overline{\mathbf{X}}_{m} \triangleq \mathbf{F}_{N}^{H} \operatorname{diag}\left(\underline{\mathbf{x}}_{m}\right) \mathbf{F}_{N, L}, \mathbf{F}_{N, L} \in \mathbb{C}^{N \times L}$ denotes the first $L$ columns of $\mathbf{F}_{N}$ and $\overline{\mathbf{Z}}_{m}$ contains noise and interferences from other targets in $\mathcal{S}$. According to 9 , the frequency-domain radar channels have the form

$$
\mathbf{F}_{N, L} \mathbf{H}=\bar{\alpha} \mathbf{b}(\tau) \mathbf{c}^{H}(\nu)
$$

${ }^{3}$ To prevent performance degradation at low SNRs due to spurious peaks and misidentification of signal and noise subspaces, improved versions of MUSIC can be employed, e.g., [27]. with $\bar{\alpha} \triangleq \alpha \mathbf{a}_{\mathrm{T}}^{T}(\widehat{\theta}) \mathbf{f}_{\mathrm{T}}$ representing the complex channel gain including the transmit beamforming effect.

Remark 1 (Duality Between OFDM Communications and OFDM Radar in (14)). Based on the observation that radar targets can be interpreted as uncooperative users from a communications perspective (as they transmit information to the radar receiver via reflections in an unintentional manner [1]4]), we point out an interesting duality between the OFDM radar signal model with ICI in (14) and an OFDM communications model with CFO (e.g., [17, Eq. (5)] and [28 Eq. (4)]). Precisely, $\mathbf{D}(\nu)$ represents CFO between the OFDM transmitter and receiver for a communications setup, while it quantifies the ICI effect due to high-speed targets for OFDM radar. Similarly, $\overline{\mathbf{X}}_{m}$ represents data/pilot symbols for communications and probing signals for radar $^{4}$. In addition, $\mathbf{h}_{m}$ represents the time-domain channel for communications and the structured (delay-Doppler parameterized) channel for radar.

In light of Remark 1, we re-formulate the radar delay-Doppler estimation problem as a communication channel estimation problem, where the objective is to jointly estimate the unstructured timedomain channels $\mathbf{H}$ and the $\mathrm{CFO} \nu$ from (14). To perform channel estimation in (14, we propose an APES-like beamformer [20]

$$
\begin{aligned}
\min _{\mathbf{w}, \mathbf{H}, \nu} & \sum_{m=0}^{M-1}\left\|\overline{\mathbf{Y}}_{m} \mathbf{w}^{*}-\mathbf{D}(\nu) \overline{\mathbf{X}}_{m} \mathbf{h}_{m}\right\|^{2} \\
\text { s.t. } & \mathbf{w}^{H} \mathbf{a}_{\mathrm{R}}(\widehat{\theta})=1
\end{aligned}
$$

where $\mathbf{w} \in \mathbb{C}^{N_{\mathrm{R}} \times 1}$ is the APES spatial beamforming vector for an estimated angle $\widehat{\theta} \in \mathcal{S}$. The optimal channel estimate for the $m^{\text {th }}$ symbol in 16 for a given $\mathrm{w}$ and $\nu$ is given by

$$
\widehat{\mathbf{h}}_{m}=\left(\overline{\mathbf{X}}_{m}^{H} \overline{\mathbf{X}}_{m}\right)^{-1} \overline{\mathbf{X}}_{m}^{H} \mathbf{D}^{H}(\nu) \overline{\mathbf{Y}}_{m} \mathbf{w}^{*} .
$$

Plugging (17) back into 16 yields

$$
\min _{\mathbf{w}, \nu} \mathbf{w}^{T} \mathbf{Q}(\nu) \mathbf{w}^{*} \quad \text { s.t. } \mathbf{w}^{H} \mathbf{a}_{\mathbf{R}}(\widehat{\theta})=1
$$

where $\mathbf{Q}(\nu) \triangleq \mathbf{R}-\boldsymbol{\Sigma}(\nu)$ is the residual SCM, $\mathbf{R}$ is the SCM of the observed data cube in 10 and

$$
\boldsymbol{\Sigma}(\nu) \triangleq \sum_{m=0}^{M-1}\left(\boldsymbol{\Pi}_{\overline{\mathbf{x}}_{m}} \mathbf{D}^{H}(\nu) \overline{\mathbf{Y}}_{m}\right)^{H}\left(\boldsymbol{\Pi}_{\overline{\mathbf{x}}_{m}} \mathbf{D}^{H}(\nu) \overline{\mathbf{Y}}_{m}\right)
$$

is the SCM of the CFO compensated observed data component that lies in the subspace spanned by the columns of the pilot symbols. For a given $\mathrm{CFO} \nu$, the optimal beamformer in (18) can be obtained in closed-form as [20]

$$
\widehat{\mathbf{w}}=\frac{\mathbf{Q}^{*}(\nu)^{-1} \mathbf{a}_{\mathrm{R}}(\widehat{\theta})}{\mathbf{a}_{\mathrm{R}}^{H}(\widehat{\theta}) \mathbf{Q}^{*}(\nu)^{-1} \mathbf{a}_{\mathrm{R}}(\widehat{\theta})} .
$$

Substituting (20) into (18), the CFO can be estimated as

$$
\widehat{\nu}=\arg \max _{\nu} \mathbf{a}_{\mathrm{R}}^{H}(\widehat{\theta}) \mathbf{Q}^{*}(\nu)^{-1} \mathbf{a}_{\mathrm{R}}(\widehat{\theta}) .
$$

Finally, plugging (20) and 21) into (17), the channel estimates can be expressed as

$$
\widehat{\mathbf{h}}_{m}=\frac{\left(\overline{\mathbf{X}}_{m}^{H} \overline{\mathbf{X}}_{m}\right)^{-1} \overline{\mathbf{X}}_{m}^{H} \mathbf{D}^{H}(\widehat{\nu}) \overline{\mathbf{Y}}_{m} \mathbf{Q}(\widehat{\nu})^{-1} \mathbf{a}_{\mathrm{R}}^{*}(\widehat{\theta})}{\mathbf{a}_{\mathrm{R}}^{T}(\widehat{\theta}) \mathbf{Q}(\widehat{\nu})^{-1} \mathbf{a}_{\mathrm{R}}^{*}(\widehat{\theta})} .
$$

\footnotetext{
${ }^{4}$ For radar sensing, every symbol acts as a pilot due to dual-functional operation on a single hardware platform.
} 


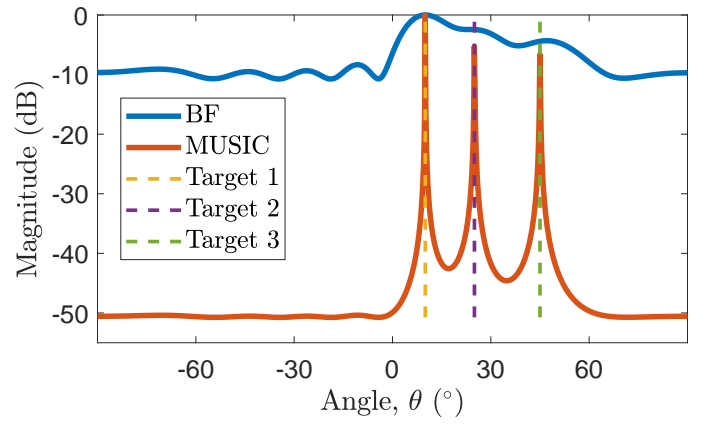

Fig. 2. MUSIC spatial spectrum of OFDM radar in Step 1 along with the results of ordinary beamforming (BF) $\mathbf{a}_{\mathrm{R}}^{T}(\theta) \mathbf{R a}_{\mathrm{R}}^{*}(\theta)$.

\subsection{Step 3: Delay-Doppler Recovery from Unstructured Chan-} nel Estimates

Given the unstructured channel estimates $\widehat{\mathbf{H}} \triangleq\left[\widehat{\mathbf{h}}_{0}, \ldots, \widehat{\mathbf{h}}_{M-1}\right]$ obtained in 22), we aim to estimate channel gain, delay and Doppler shift via a least-squares (LS) approach by exploiting the structure in 15) as follows:

$$
\min _{\alpha, \tau, \nu}\left\|\mathbf{F}_{N, L} \widehat{\mathbf{H}}-\bar{\alpha} \mathbf{b}(\tau) \mathbf{c}^{H}(\nu)\right\|_{F}^{2} .
$$

In 23, delay and Doppler estimates $\widehat{\tau}$ and $\widehat{\nu}$ can be obtained simply via 2-D FFT (i.e., IFFT and FFT across the columns and rows of $\mathbf{F}_{N, L} \widehat{\mathbf{H}}$, respectively). Then, channel gain can be estimated as $\widehat{\bar{\alpha}}=$ $\mathbf{b}^{H}(\widehat{\tau}) \mathbf{F}_{N, L} \widehat{\mathbf{H}} \mathbf{c}(\widehat{\nu}) /\left(\|\mathbf{b}(\widehat{\tau})\|^{2}\|\mathbf{c}(\widehat{\nu})\|^{2}\right)$. The overall algorithm is summarized in Algorithm 1

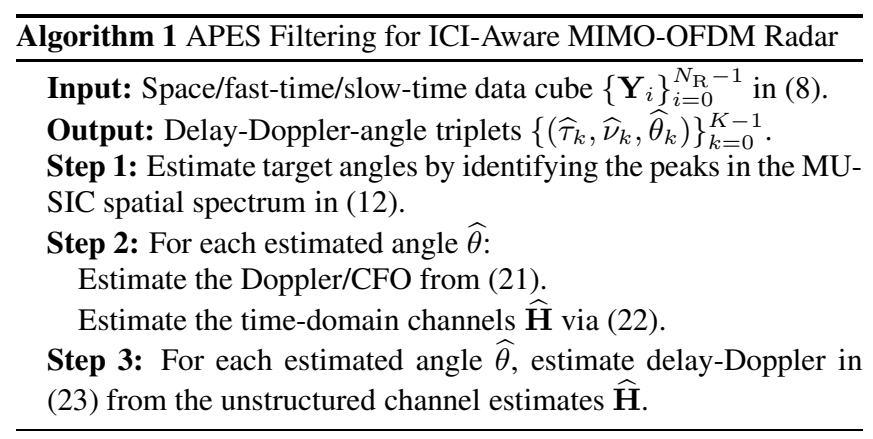

\section{SIMULATION RESULTS}

To demonstrate the performance of Algorithm 1 we consider an OFDM system with $f_{c}=60 \mathrm{GHz}, B=50 \mathrm{MHz}, N=2048$, $\Delta f=24.41 \mathrm{kHz}, T_{\mathrm{sym}}=51.2 \mu \mathrm{s}, M=64, N_{\mathrm{T}}=8$ and $N_{\mathrm{R}}=8$. The data symbols $\mathbf{X}$ are randomly generated from the QPSK alphabet and the transmit beamformer is set to point towards $30^{\circ}$, i.e., $\mathbf{f}_{\mathrm{T}}=\mathbf{a}_{\mathrm{T}}^{*}\left(30^{\circ}\right)$. To illustrate the output of Algorithm 1 we first consider a scenario consisting of $K=3$ targets with the ranges $(60,100,150) \mathrm{m}$, the velocities $(-60,30,120) \mathrm{m} / \mathrm{s}$, the angles $\left(10^{\circ}, 25^{\circ}, 45^{\circ}\right)$ and the SNRs (i.e., $\left.\left|\alpha_{k}\right|^{2} / \sigma^{2}\right)(30,15,25) \mathrm{dB}$. Fig. 2 shows the MUSIC spectrum (12) obtained in Step 1 of Algorithm 1 In addition, Fig. 3 demonstrates the range-velocity profiles obtained in Step 3 for each target angle along with the results of standard 2-D FFT [9]. It is observed that the proposed algorithm can successfully separate multiple target reflections in the angular domain, estimate their Dopplers/CFOs for ICI elimination and accurately recover delays and Dopplers.

Second, we compare the performance of Algorithm 1 with the 2-D FFT benchmark [9] in a single-target scenario with $(R, v, \theta)=$
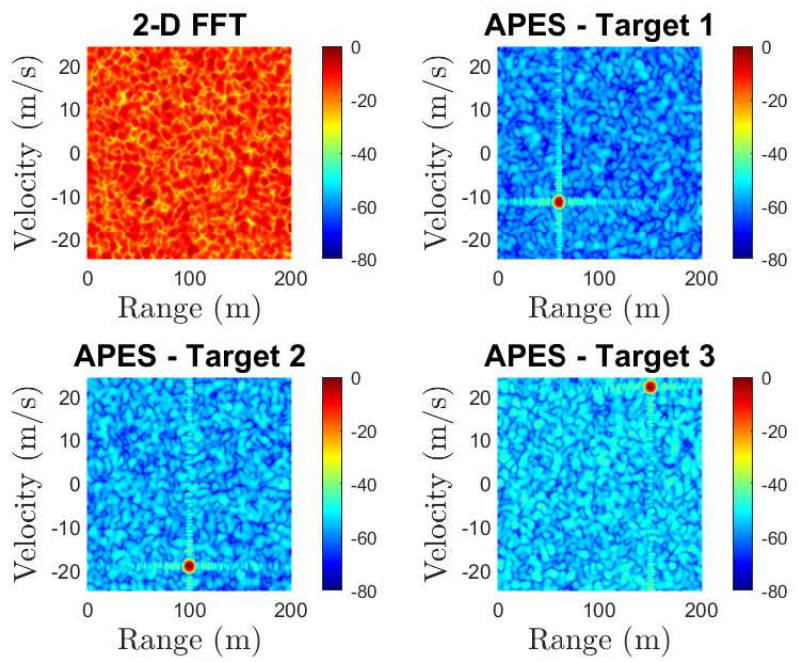

Fig. 3. Range-velocity profiles obtained by standard 2-D FFT after receive beamforming towards $10^{\circ}$ and those obtained by Algorithm 1 in Step 3 as the output of 2-D FFT of target-specific frequency-domain channel estimates for Target 1, Target 2 and Target 3.
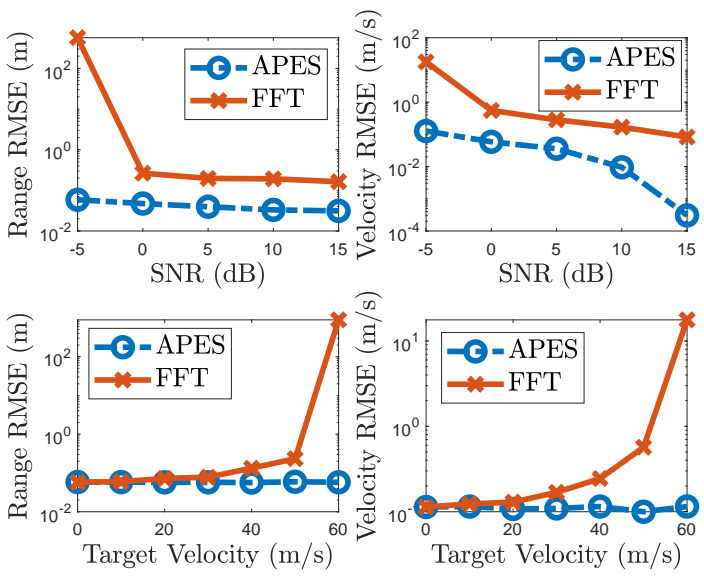

Fig. 4. Range and velocity estimation performances of Algorithm 1 and 2-D FFT benchmark.

$\left(80 \mathrm{~m}, 70 \mathrm{~m} / \mathrm{s}, 30^{\circ}\right)$ and an SNR of $-5 \mathrm{~dB}$ using $M=8$ symbols. Since there exists no previous estimators for ICI-aware MIMOOFDM radar, 2-D FFT is applied on the fast-time/slow-time snapshot obtained by receive beamforming of the data cube towards the true target angle. Fig. 4 shows the range and velocity RMSEs of Algorithm 1 and the 2-D FFT benchmark with respect to SNR and target velocity over 100 Monte Carlo noise realizations. As seen from the figure, ICI-induced high side-lobe levels in the delay-Doppler domain significantly degrade the performance of the 2-D FFT algorithm, while the proposed algorithm can suppress the ICI effect by exploiting the signal structure within an APES beamforming framework.

\section{CONCLUSION}

This paper addresses the parameter estimation problem for a MIMOOFDM radar in the presence of non-negligible ICI caused by highmobility targets. Based on an APES spatial filtering approach, a novel delay-Doppler-angle estimation algorithm is proposed by reformulating radar sensing as a communication channel estimation problem. Simulation results show that the proposed algorithm enables high-accuracy multi-target parameter estimation under strong ICI by separating individual target reflections in the angular domain. 


\section{REFERENCES}

[1] F. Liu, C. Masouros, A. Petropulu, H. Griffiths, and L. Hanzo, "Joint radar and communication design: Applications, stateof-the-art, and the road ahead," IEEE Transactions on Communications, pp. 1-1, 2020.

[2] K. V. Mishra, M. R. Bhavani Shankar, V. Koivunen, B. Ottersten, and S. A. Vorobyov, "Toward millimeter-wave joint radar communications: A signal processing perspective," IEEE Signal Processing Magazine, vol. 36, no. 5, pp. 100-114, Sep. 2019.

[3] L. Zheng, M. Lops, Y. C. Eldar, and X. Wang, "Radar and communication coexistence: An overview: A review of recent methods," IEEE Signal Processing Magazine, vol. 36, no. 5, pp. 85-99, 2019.

[4] Alex R Chiriyath, Bryan Paul, and Daniel W Bliss, "Radarcommunications convergence: Coexistence, cooperation, and co-design," IEEE Transactions on Cognitive Communications and Networking, vol. 3, no. 1, pp. 1-12, 2017.

[5] Dingyou Ma, Nir Shlezinger, Tianyao Huang, Yimin Liu, and Yonina C Eldar, "Joint radar-communication strategies for autonomous vehicles: Combining two key automotive technologies," IEEE Signal Processing Magazine, vol. 37, no. 4, pp. 85-97, 2020.

[6] C. Aydogdu, M. F. Keskin, G. K. Carvajal, O. Eriksson, H. Hellsten, H. Herbertsson, E. Nilsson, M. Rydstrom, $\mathrm{K}$. Vanas, and H. Wymeersch, "Radar interference mitigation for automated driving: Exploring proactive strategies," IEEE Signal Processing Magazine, vol. 37, no. 4, pp. 72-84, 2020.

[7] A. Hassanien, M. G. Amin, E. Aboutanios, and B. Himed, "Dual-function radar communication systems: A solution to the spectrum congestion problem," IEEE Signal Processing Magazine, vol. 36, no. 5, pp. 115-126, Sep. 2019.

[8] F. Liu, L. Zhou, C. Masouros, A. Li, W. Luo, and A. Petropulu, "Toward dual-functional radar-communication systems: Optimal waveform design," IEEE Transactions on Signal Processing, vol. 66, no. 16, pp. 4264-4279, Aug 2018.

[9] C. Sturm and W. Wiesbeck, "Waveform design and signal processing aspects for fusion of wireless communications and radar sensing," Proceedings of the IEEE, vol. 99, no. 7, pp. 1236-1259, July 2011.

[10] M. Bică and V. Koivunen, "Generalized multicarrier radar: Models and performance," IEEE Transactions on Signal Processing, vol. 64, no. 17, pp. 4389-4402, Sep. 2016.

[11] Fuqiang Zhang, Zenghui Zhang, Wenxian Yu, and Trieu-Kien Truong, "Joint range and velocity estimation with intrapulse and intersubcarrier Doppler effects for OFDM-based RadCom systems," IEEE Transactions on Signal Processing, vol. 68, pp. 662-675, 2020.

[12] R. F. Tigrek, W. J. A. De Heij, and P. Van Genderen, "OFDM signals as the radar waveform to solve Doppler ambiguity," IEEE Transactions on Aerospace and Electronic Systems, vol. 48, no. 1, pp. 130-143, Jan 2012.

[13] Sayed Hossein Dokhanchi, Bhavani Shankar Mysore, Kumar Vijay Mishra, and Björn Ottersten, "A mmWave automotive joint radar-communications system," IEEE Transactions on Aerospace and Electronic Systems, vol. 55, no. 3, pp. 12411260, 2019.
[14] G. Hakobyan, M. Ulrich, and B. Yang, "OFDM-MIMO radar with optimized nonequidistant subcarrier interleaving," IEEE Transactions on Aerospace and Electronic Systems, vol. 56, no. 1, pp. 572-584, 2020.

[15] Gor Hakobyan and Bin Yang, "A novel intercarrierinterference free signal processing scheme for OFDM radar," IEEE Transactions on Vehicular Technology, vol. 67, no. 6, pp. 5158-5167, 2017.

[16] Jinsoo Lim, Sung-Rae Kim, and Dong-Joon Shin, “Two-step Doppler estimation based on intercarrier interference mitigation for OFDM radar," IEEE Antennas and Wireless Propagation Letters, vol. 14, pp. 1726-1729, 2015.

[17] Yinghao Ge, Weile Zhang, Feifei Gao, and Hlaing Minn, "Angle-domain approach for parameter estimation in highmobility OFDM with fully/partly calibrated massive ULA," IEEE Transactions on Wireless Communications, vol. 18, no. 1, pp. 591-607, 2018.

[18] Ralph Schmidt, "Multiple emitter location and signal parameter estimation," IEEE transactions on antennas and propagation, vol. 34, no. 3, pp. 276-280, 1986.

[19] K. Luo and A. Manikas, "Superresolution multitarget parameter estimation in MIMO radar," IEEE Transactions on Geoscience and Remote Sensing, vol. 51, no. 6, pp. 3683-3693, 2013.

[20] Luzhou Xu, Jian Li, and Petre Stoica, "Target detection and parameter estimation for MIMO radar systems," IEEE Transactions on Aerospace and Electronic Systems, vol. 44, no. 3, pp. 927-939, 2008.

[21] Y. L. Sit, B. Nuss, and T. Zwick, "On mutual interference cancellation in a MIMO OFDM multiuser radar-communication network," IEEE Transactions on Vehicular Technology, vol. 67, no. 4, pp. 3339-3348, April 2018.

[22] Martin Braun, "OFDM radar algorithms in mobile communication networks," Karlsruher Institutes für Technologie, 2014.

[23] P. Kumari, J. Choi, N. González-Prelcic, and R. W. Heath, "IEEE 802.11ad-based radar: An approach to joint vehicular communication-radar system," IEEE Transactions on Vehicular Technology, vol. 67, no. 4, pp. 3012-3027, April 2018.

[24] Y. . Liang, R. Schober, and W. Gerstacker, "Time-domain transmit beamforming for MIMO-OFDM systems with finite rate feedback," IEEE Transactions on Communications, vol. 57, no. 9, pp. 2828-2838, 2009.

[25] Timo Roman, Samuli Visuri, and Visa Koivunen, "Blind frequency synchronization in OFDM via diagonality criterion," IEEE Transactions on Signal Processing, vol. 54, no. 8, pp. 3125-3135, 2006.

[26] Y. Ge, W. Zhang, F. Gao, and G. Y. Li, "Frequency synchronization for uplink massive MIMO with adaptive MUI suppression in angle domain," IEEE Transactions on Signal Processing, vol. 67, no. 8, pp. 2143-2158, 2019.

[27] Michael L McCloud and Louis L Scharf, "A new subspace identification algorithm for high-resolution DOA estimation," IEEE Transactions on Antennas and Propagation, vol. 50, no. 10, pp. 1382-1390, 2002.

[28] Weile Zhang, Qinye Yin, and Wenjie Wang, "Blind closedform carrier frequency offset estimation for OFDM with multiantenna receiver," IEEE Transactions on Vehicular Technology, vol. 64, no. 8, pp. 3850-3856, 2014. 